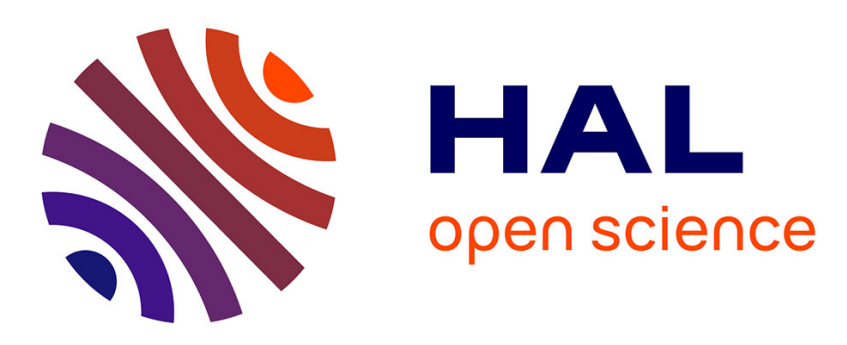

\title{
Perturbations of the flow induced by a microcapsule in a capillary tube
}

Jonathan Gubspun, Marc Georgelin, Julien Deschamps, Marc Leonetti, Clément de Loubens, Roberto Trozzo

\section{- To cite this version:}

Jonathan Gubspun, Marc Georgelin, Julien Deschamps, Marc Leonetti, Clément de Loubens, et al.. Perturbations of the flow induced by a microcapsule in a capillary tube. Fluid Dynamics Research, 2017, 49 (3), pp.035501. 10.1088/1873-7005/aa6270 . hal-02020111

\section{HAL Id: hal-02020111 \\ https://hal.science/hal-02020111}

Submitted on 23 Oct 2019

HAL is a multi-disciplinary open access archive for the deposit and dissemination of scientific research documents, whether they are published or not. The documents may come from teaching and research institutions in France or abroad, or from public or private research centers.
L'archive ouverte pluridisciplinaire HAL, est destinée au dépôt et à la diffusion de documents scientifiques de niveau recherche, publiés ou non, émanant des établissements d'enseignement et de recherche français ou étrangers, des laboratoires publics ou privés. 


\title{
Perturbations of the flow induced by a microcapsule in a capillary tube
}

\author{
J. Gubspun, M. Georgelin, J. Deschamps, and M. Leonetti* \\ Aix-Marseille Université, CNRS, Centrale Marseille, IRPHE, UMR7342, 13384, Marseille, France \\ C. de Loubens \\ Universite Grenoble Alpes, LRP, F-38000 Grenoble, France and \\ CNRS, LRP, F-38000 Grenoble, France \\ R. Trozzo \\ Aix-Marseille Université, CNRS, Centrale Marseille, M2P2, UMR7430, 13451, Marseille, France \\ F. Edwards-Levy \\ Institut de Chimie Moléculaire de Reims, UMR7312, \\ CNRS-Université de Reims Champagne Ardenne, 51100 Reims, France
}

(Dated: October 23, 2019)

\begin{abstract}
Soft microcapsules moving in a cylindrical capillary deform from quasi-spherical shapes to elongated shapes with an inversion of curvature at the rear. We investigated the perturbation of the flow by particle tracking velocimetry around deformed microcapsules in confined flow. These experiments are completed by numerical simulations. Microcapsules are made of a thin membrane of polymerized human albumin and their shear elastic moduli are previously characterized in a cross flow chamber. Firstly, the velocity of the microcapsule can be calculated by theoretical predictions for rigid spheres, even for large deformations as 'parachute-like' shapes, if a relevant definition of the ratio of confinement is chosen. Secondly, at the rear and the front of the microcapsule, the existence of multiple recirculation regions is governed by the local curvature of the membrane. The amplitudes of these perturbations increase with the microcapsule deformation, whereas their axial extents are comparable to the radius of the capillary whatever the confinement and the capillary number. We conclude that whereas the motion of microcapsules in confined flow has quantitative similitudes with rigid spheres in term of velocity and axial extent of the perturbation, their presence induces variations in the flow field that are related to the local deformation of the membrane as in droplets.
\end{abstract}

\section{INTRODUCTION}

Controlling the delivery of molecules is of prime importance in various domains as medicine, biology, nutrition or cosmetics. Microencapsulation of active materials within a sub-millimetric shell is a promising technique to take up this challenge $[1,2]$. For example, encapsulation has allowed workers to develop new treatments of chronic heart disease by controlling the spatio-temporal release of growth factors in the blood stream [3] or to create artificial red blood cells [4]. The delivery is achieved by hydrodynamic stresses or external stimuli inducing dynamic of deformations of the microcapsule and transfer through its membrane or even its breakdown [5]. The mechanical properties of the membrane have so to be tuned according to the hydrodynamic conditions encountered in the final application.

Microcapsule membranes can be made of cross-linked polymer [6] or complex of polyelectrolytes [7, 8]. These membranes show a variety of elastic behaviors that can be varied with the kind of constituents and their

\footnotetext{
* leonetti@irphe.univ-mrs.fr
}

concentration [9]. Polysiloxane [10] and nylon microcapsules [11, 12] break for minute deformation while Human Serum Albumin (HSA) microcapsules can be deformed elastically up to $180 \%$ of stretching [13], for example. Up to now, experimental efforts have been made to characterize accurately the elastic properties of the membrane. Compression between two plates [14] and simple flows as elongation $[9,11]$, shear $[12,15,16]$, Poiseuille [17, 18], flow through constriction [19] or centrifugal flow [20] have been used to deform microcapsules and measure their shear elastic modulus in the small deformation regime and moderate in the case of Poiseuille flow. More recently, the non-linear elastic model and the viscoelastic properties of the membrane were deduced in the regime of large deformations [13] and from the dynamic of tank-treading in simple shear flow [21] for HSA microcapsules. Numerical simulations play a major role to identify the relevant mechanical properties of the membrane [22] as its constitutive law $[13,23]$, its viscosity [24] or its bending modulus [25]. Experimental and numerical studies on microcapsules in flow have focused a strong attention to the shape and its dynamics but not on the flow around it and notably on the perturbation of the applied flow. However, it plays a major role to understand the interactions between several soft particles in a three-dimensional suspension [26] 
but also in a confined channel [27]. These perturbations can have dramatic effects on the formation of clusters as observed for drops [28] and biological suspensions of soft particles like blood [29].

In confined flows, microcapsules are sufficiently soft to deform and move through channel of comparable dimensions such as blood vessels. When the microcapsule is small compared to the size of the channel, its shape is slightly deformed and the flow approximately the same than a Poiseuille flow. On the contrary, when the confinement is such that the size of the object is comparable to the size of the channel or even bigger, both the flow and the microcapsule shape may be largely modified. The latter situation has been studied for bubbles, drops or solid particles. Taylor was one of the first to report the streamlines of the outer flow perturbed by the bubble [30]. Then theoretical developments have been conducted for solid particles when immersed in the center or off-center of a Poiseuille flow [31, 32] and also quasi-spherical drops [33-35]. Ho \& Leal observed experimentally recirculation inside a drop and the existence of stagnation rings on its front and rear [36]. The axisymmetric recirculation of the fluid has also been studied between two adjacent drops spaced by a typical distance of the same order than the drop size inside the capillary. This analysis has been extended numerically for different capillary numbers, viscosities and confinements with similar qualitative results [37].

Whereas the flow has been studied around simple objects such as droplets or bubbles, no detailed investigations have been reported regarding the flow generated by soft particles as microcapsules with a comparison between experimental, analytical and numerical results. While the shear is transmitted across the fluid interface of bubbles and droplets, the solid membrane of microcapsules should induce major differences in term of fluid-structure interactions. Moreover, the deformation of microcapsules in narrow tube presents an inversion of the membrane curvature at its rear, called 'parachutelike' shape in the studies on vesicles [38-42] and capsules $[17,43,44]$, whereas previous studies on drops and bubbles [30, 33-37, 45] have not considered this situation $[46,47]$.

In this paper, we study the effects of deformable microcapsules on the surrounding flow in a capillary channel. After mechanical characterization using an elongation flow, a successive approach is developed: i) determination of several families of characteristic shapes of capsules in capillaries varying the capillary number and the confinement and ii) determination of the flow field around these microcapsules measured by microparticles tracking velocimetry (PTV). We study microcapsules made of cross-linked proteins (HSA), the only ones which have been fully characterized: constitutive law in all the regimes of deformation, Poisson coefficient, shear elastic modulus and shear membrane viscosity. To discuss the results on the measured flow, numerical simulations are performed using the same deformations and microcapsule velocity as in experiments to calculate the flow field using the boundary element method for Stokes flow conditions. Note that we don't consider the full fluid-structure interaction but underformable axisymmetric solid body with no slip velocity at the membrane. We analyze the evolution of the velocity of the microcapsule with its deformation. The topology of the perturbation of flow field is emphasized according to the curvature of the rear of the particle, as the amplitude and spatial extent of this perturbation.

\section{MATERIALS AND METHODS}

\section{A. Microcapsule preparation}

Microcapsules were prepared by interfacial crosslinking of human serum albumin (HSA) with terephthaloyl chloride (see Andry et al. [6] for details). HSA, organic solvents (chloroform and cyclohexane), crosslinking agent (terephtaloyl chloride) and surfactants (sorbitan trioleate and polysorbate) were purchased from LFB Biomedicaments, SDF, Acros Organics and Sigma, respectively. HSA was dissolved in a $\mathrm{pH} 5.9$ phosphate buffer at the concentration $7.5 \mathrm{~g} / 100 \mathrm{~mL}$. This aqueous solution was emulsified in cyclohexane containing $2 \%$ $(\mathrm{w} / \mathrm{v})$ sorbitan trioleate at a stirring speed of $625 \mathrm{rpm}$. A $2.5 \%(\mathrm{w} / \mathrm{v})$ solution of terephthaloyl chloride in chloroform:cyclohexane $(1: 4 \mathrm{v} / \mathrm{v})$ was then added to the emulsion and the cross-linking reaction was allowed to develop for $30 \mathrm{~min}$. The reaction was stopped by dilution of the reaction medium with cyclohexane. The microcapsules were separated from the organic phase by centrifugation and washed successively with cyclohexane, with water containing $2 \%(\mathrm{w} / \mathrm{v})$ polysorbate and finally thrice with pure water. Microcapsules were then diluted of about thousand times and mixed very gently for at least 24 hours in $98 \%$ glycerol (VWR) to allow the internal phase to equilibrate with the external one by diffusion through the pores of the membrane. The membrane is permeable on long time scale but may be considered as impermeable on short time scale, namely the volume is conserved during the experiments as shown in [48].

Finally, we obtain a diluted suspension of spherical microcapsules whose radii range from 120 to $230 \mu \mathrm{m}$, embedded in glycerol.

\section{B. Mechanical characterization of microcapsule with elongation flow}

Beyond their softness, the main advantage of viscoelastic membranes made of human albumin compared to others (ovalbumin, alginate, alginate-albumin mixing for example) is their full characterization supporting by previous studies from the small to large deformations providing the constitutive law [13]. The membrane mechanical 



FIG. 1. Up - Schematic diagram of the experimental setup with an elongation flow: a microfluidic cross chamber. Bottom - Example of the deformation of a capsule with an initial radius of $37 \mu \mathrm{m}$. The variation of the deformation with the elongation shear stress provides the shear elastic modulus. See text and [9] for details.

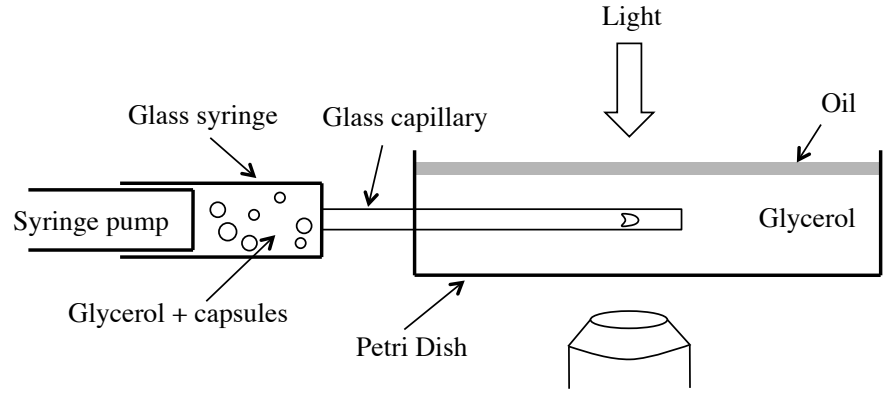

Microscope objective

FIG. 2. Schematic diagram of the experimental set-up. HSA microcapsules (120 to $230 \mu \mathrm{m}$ radius) are in suspension in a solution of glycerol and pushed by a syringe pump in a capillary tube (150 to $200 \mu \mathrm{m}$ inner radius). The glycerol is seeded with particles to characterize the flow around the microcapsules by PTV. Observations are made with an inverted microscope and a high speed camera.

response is in excellent agreement with the generalized Hooke law with a 2D Poisson coefficient $\nu_{s}$ equal to 0.4. Viscous dissipation inside the human albumin membrane determined in [21] is not relevant in the present paper as we study axisymmetric stationary shapes without inter- facial flow.

We measure separately the evolution of $G_{s}$ with the radius $a$ by studying the deformations of several microcapsules under a planar elongation flow in the linear regime of deformations with the same technique described in [9]. As shown in $[9,13,48]$, the shear elastic modulus $G_{s}$ of HSA microcapsules increases with the radius. The size dependance of $G_{s}$ has been explained in considering that all HSA molecules initially available in the primary droplet, finally are cross-linked in the capsule membrane [48]. For the parameters of microcapsule preparation given above, $G_{s}$ increases from $0.05 \mathrm{~N} / \mathrm{m}$ to $0.5 \mathrm{~N} / \mathrm{m}$ when $a$ increases from 120 to $230 \mu \mathrm{m}$. The evolution of $G_{s}$ with the radius can be fitted by an exponential law:

$$
G_{s}=1.9 \times 10^{-3} \exp \left(a \times 2.6 \times 10^{-2}\right)
$$

with the radius $a$ in $\mu \mathrm{m}$ and the shear elastic modulus $G_{s}$ in $\mathrm{N} / \mathrm{m}$. The dilatation elastic coefficient $\mathrm{K}$ can be deduced with the equation $K=G_{s}\left(1+\nu_{s}\right) /\left(1-\nu_{s}\right)$ with $\nu_{s}$ equal to 0.4 [21] . Equation 1 allows to determine the capillary number which evaluates the hydrodynamic shear stress at membrane versus the elastic response. With it and the confinement, readers have all the necessary parameters to perform new numerical studies with different constitutive laws and compare to our experimental shapes and capsule velocity.

\section{Experimental setup for Poiseuile flow}

An axisymmetric Poiseuille flow is generated in glass capillary tubes (Hampton research) of $80 \mathrm{~mm}$ length and 150 to $200 \mu \mathrm{m}$ nominal inner radii $R$. Glass tubes are glued on steel needles (Harvard apparatus) and plugged to a $10 \mathrm{~mL}$ glass syringe (Poulten and Graph $\mathrm{GmbH}$ ). The glycerol with microcapsules is pushed in the capillary tube with an home made syringe pump designed with an actuator PI 235.52S. The glass tube is immersed in a Petri dish full of glycerol to match the optical index of its inner and outer media and prevent any optical distortion (Fig. 2). A thin layer of oil covers the glycerol to prevent water absorption. The entire system is rigid to reach in less than $100 \mathrm{~ms}$ a stationary flow in the capillary. The Petri dish is mounted on top of an inverted microscope Olympus IX70. Microcapsules are observed in the midplane of the channel with a magnification $\times 10$ $(1.70 \mu \mathrm{m} / \mathrm{pix})$ and a high speed camera Photron SA3 (5000 i/s, $1024 \times 512$ pixels).

In the reference frame of the microcapsule and far from it, $\mathbf{V}$ is axisymmetric and satisfies the Poiseuille equation:

$$
\mathbf{V}(r)=\left(2 \bar{V}\left(1-\frac{r^{2}}{R^{2}}\right)-V_{\text {caps }}\right) \mathbf{e}_{\mathbf{z}}
$$

where $\bar{V}$ is the mean flow velocity, $V_{\text {caps }}$ the capsule velocity. $r$ and $z$ are the radial and axial coordinates, $\mathbf{e}_{\mathbf{r}}$ and $\mathbf{e}_{\mathbf{z}}$ the respective unit vectors (Fig. 3). 


\section{Flow measurements \& numerical simulations}

For each microcapsule, initial size and capillary radius are measured at rest. As the microcapsule is flowing in the tube we extract the profile of its stationary deformed shape in the plane $\left(\mathbf{e}_{\mathbf{r}}, \mathbf{e}_{\mathbf{z}}\right)$ and measure the radial extension of the microcapsule $L_{r}$, the position of its center of mass and its velocity $V_{\text {caps }}$ (Fig. 3). $V_{\text {caps }}$ is measured by tracking the front of the microcapsule in the reference frame of the laboratory and ranges from 0.5 to $20 \mathrm{~mm} / \mathrm{s}$.
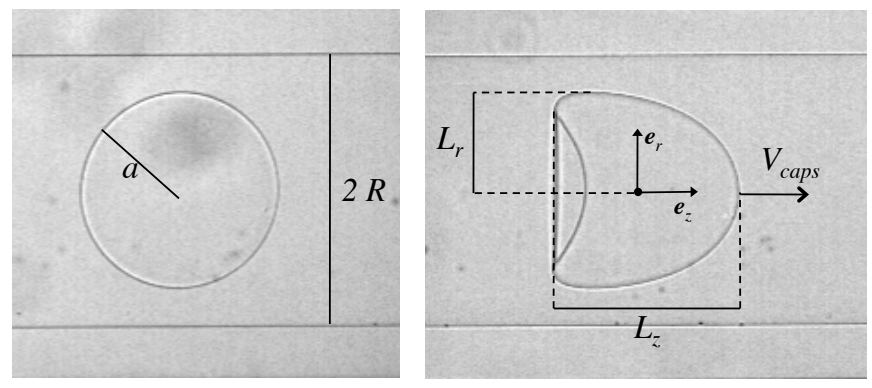

FIG. 3. Parameters used to characterize the shape of microcapsules at rest and under flow. The geometry of the capillary tube is axisymmetric $\left(\mathbf{e}_{\mathbf{r}}, \mathbf{e}_{\mathbf{z}}\right)$. The radii of the microcapsule $a$ and the capillary tube $R$ are measured at rest (left). The velocity $V_{\text {caps }}$, radial extension $L_{r}$, axial extension $L_{z}$ and center of mass of the deformed microcapsule are measured under flow (right).

The flow in presence of isolated microcapsules is characterized by Particle Tracking Velocimetry (PTV). Polystyrene microspheres (Bangs Laboratories Inc.) with a radius ranging from 8 to $16 \mu \mathrm{m}$ are seeded in glycerol at a concentration of $2.4 \mathrm{mg} / \mathrm{mL}$. The size, very small compared to capsule radius has also been chosen to be detectable by PTV without measurable effect on the flow. The x10 objective has a large depth of field and integrates particles on a layer which is approximately $25 \%$ of the diameter of the channel. All visible particles are taken into account to characterize the flow. Moreover the microcapsule move too quickly ( $V_{\text {caps }}$ up to $20 \mathrm{~mm} / \mathrm{s}$ ) to track the capsule along the tube. In these conditions, the entire velocity field cannot be determined in the reference frame of the laboratory by one experiment. Thus to obtain the full velocity field around the microcapsule it is necessary to stack partial informations field extracted from several observations of the same microcapsule in the same conditions. Thus the microcapsule is pushed and sucked back up to 10 times in the capillary for a given flow rate to reconstruct the flow field in its reference frame. Particles are tracked and trajectories are extracted with Matlab (MathWorks). Finally, all the trajectories are stacked together to calculate the velocities in the axial $v_{z}$ and radial $v_{r}$ directions over 20 boxes along $\mathbf{e}_{\mathbf{r}}$ and 40 along $\mathbf{e}_{\mathbf{z}}$ (Fig. 4).

The microcapsule velocity in a capillary with a flow rate $\pi R^{2} \bar{V}$ has been measured for 21 microcapsules. Each microcapsule is observed for different flow rates. The mean velocity $\bar{V}$ of the fluid in the capillary is deduced from the flow rate applied by the syringe pump or measured by tracking the fastest particles. Both methods were compared and match perfectly.

Experiments were carried out at a room temperature of $22 \pm 0.5^{\circ} \mathrm{C}$ controlled inside the Petri dish with a precision of $0.1^{\circ} \mathrm{C}$. The viscosity of the glycerol $\eta$ was measured with a rheometer ThermoScientifc Haake Mars III in a cone-plate geometry $\left(2^{\circ}\right.$ cone, $60 \mathrm{~mm}$ diameter $)$ as a function of the temperature and ranged from 0.99 to 1.18 Pa.s.

The flow field is also calculated by numerical simulations by considering different shapes of microcapsules extracted from the experiments. Instead of solving the full complex fluid structure interaction problem of a deformable object in a capillary tube, the microcapsule is considered as a solid body translated in a Poiseuille flow with a velocity $V_{\text {caps }}$. Briefly, the flow is computed by the boundary element method for Stokes flow conditions $[49,50]$. The code has been validated in several configurations (axi / non-axisymmetric) in a capillary for rigid bodies and vesicles [51] and in free space for vesicles [5254].

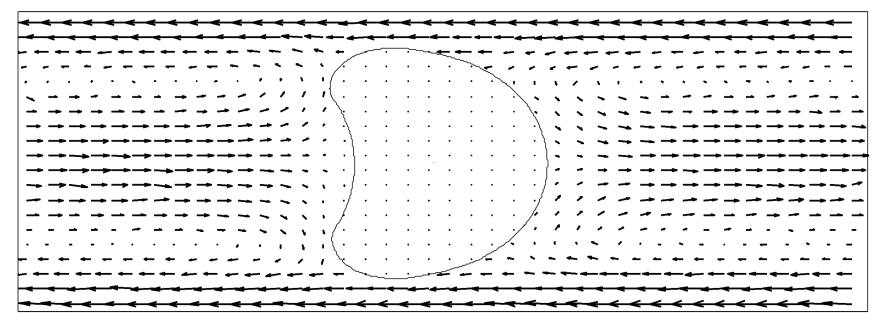

FIG. 4. Velocity field measured by PTV in the frame of reference of the microcapsule. The velocity field is measured from 7 movies of the same microcapsule pushed in the same conditions ; $a=159 \mu \mathrm{m}, 2 R=403 \mu \mathrm{m}, \beta=0.8$ (confinement, see Eq. 4$), \bar{V}=3.65 \mathrm{~mm} / \mathrm{s}, G_{s}=0.12 \mathrm{~N} / \mathrm{m}, C a=0.032$.

\section{MICROCAPSULE SHAPES IN A CAPILLARY}

The shape of microcapsules moving along capillaries varies according two dimensionless parameters [44]:

- the capillary number

$$
C a=\frac{\eta \bar{V}}{G_{s}}
$$

- the confinement ratio

$$
\beta=\frac{a}{R}
$$

While the confinement $\beta$ is a geometrical parameter, the capillary number $C a$ is the ratio of viscous stress $\eta \bar{V} / a$ on the membrane elastic response $G_{s} / a$. For 
$\beta<<1$, the shape is quasi-spherical. As the capillary number (the microcapsule velocity) increases, the curvature at the rear changes its sign with the so-called 'parachute-like' shape already observed with alginatealbumine capsules [17] but with an unknown membrane material (constitutive law), and only with macroscopic capsules (diameter $=4 \mathrm{~mm}$ ). Note also in figure 6 of [17] that all capsules are not axisymmetrical while it is the case here.

The confinement can be larger than unity contrary to a rigid particle, a scarcely studied range. The seminal work [17] focuses mainly on $\beta \leq 0.87$. For $\beta>0.9$, the lubrication film between the membrane and the wall is expected to play an important role. The array of shapes (Fig. 5) provides a good representation of all shapes which can be observed. These microcapsules are sufficiently soft to deform and enter in pores smaller than their own sizes (case $\beta>1$ ). The results show an increasing of the lubrication film thickness with $C a$ as expected from studies on droplets and red blood cells moving in a capillary [43, 55]. However, the range of data is too restricted to infer the power exponent. The mean flow velocity $\bar{V}$ (equation 2 ) is given to provide all necessary data for any numerical studies. The shear elastic modulus can be deduced from equation 1.

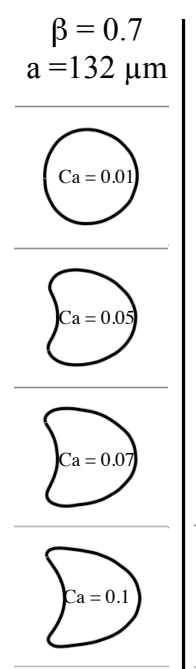

a

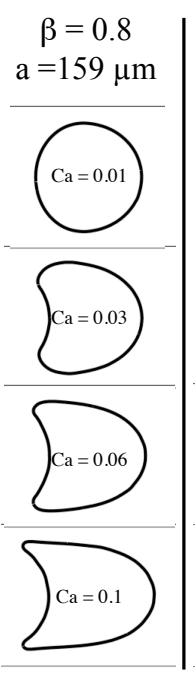

b

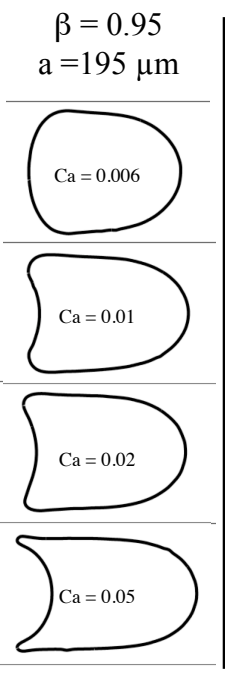

C

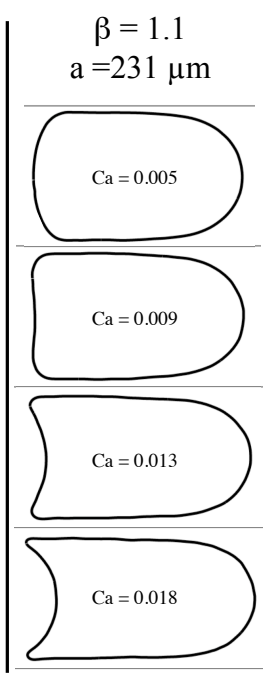

d
FIG. 5. Experimental shapes of microcapsules for different confinement ratio $\beta=a / R$ and capillary numbers $C a$. When $C a$ increases, the curvature of the microcapsule rear inverts. The width of each box is equal to the capillary diameter $2 R$ of $384 \mu \mathrm{m}$ for column a and $403 \mu \mathrm{m}$ for columns b, c and $\mathrm{d}$. Mean flow velocity $\bar{V}$ from top to bottom for each column:

a. $0.67 \mathrm{~mm} / \mathrm{s}, 2.59 \mathrm{~mm} / \mathrm{s}, 3.73 \mathrm{~mm} / \mathrm{s}, 5.91 \mathrm{~mm} / \mathrm{s}$.

b. $1.25 \mathrm{~mm} / \mathrm{s}, 3.65 \mathrm{~mm} / \mathrm{s}, 6.54 \mathrm{~mm} / \mathrm{s}, 12.7 \mathrm{~mm} / \mathrm{s}$.

c. $1.95 \mathrm{~mm} / \mathrm{s}, 4.04 \mathrm{~mm} / \mathrm{s}, 6.68 \mathrm{~mm} / \mathrm{s}, 13.92 \mathrm{~mm} / \mathrm{s}$.

d. $3.98 \mathrm{~mm} / \mathrm{s}, 6.74 \mathrm{~mm} / \mathrm{s}, 10.02 \mathrm{~mm} / \mathrm{s}, 13.72 \mathrm{~mm} / \mathrm{s}$.

\section{MICROCAPSULE VELOCITY}

We investigate the effects of flow parameters on the velocity of the capsule. Whereas the confinement ratio $\beta$ is usually defined with the shape of the capsule at rest (equation 4 ), we calculate the local confinement $L_{r} / R$ by considering the radial extension of the capsule under flow (Fig. 3). The velocity of the capsule relative to the mean flow velocity $V_{\text {caps }} / \bar{V}$ as a function of $L_{r} / R$ follows the same master curve for all the microcapsules $(\beta$ ranges from 0.35 to 1.1) and large variations of $L_{r} / R$ (from 0.3 to 0.9 , Fig. 6). It is interesting to compare this evolution with analytical or asymptotical theoretical results obtained for rigid particles in capillaries.

For small values of confinement, the microcapsule is nearly spherical. In this case, the velocity of a solid sphere of radius $a$ moving at the center of a cylindrical capillary [31] is given by:

$$
\frac{V_{\text {caps }}}{\bar{V}}=2-\frac{4}{3} \beta^{2} \quad \text { for } \beta \ll 1
$$

When $\beta$ tends to 0 , the velocity of the sphere is maximal and equal the velocity of the flow in the center of the capillary, i.e. $2 \bar{V}$. For deformable spheres, as microcapsules, if we replace $\beta$ by the local confinement under flow $L_{r} / R$ in the previous equation, we obtain an excellent agreement with experiments up to $\beta \leq 0.6$, as the shape of the microcapsules are quasi-spherical for small confinements.

As $\beta$ tends to 1 , the shape is strongly deformed even at small capillary number (or velocity) at the rear of the microcapsule but also on its lateral part which is facing the capillary wall. Then, the transverse size $L_{r}$ is smaller than the initial microcapsule size $a$ and the capillary radius $R$. As the capillary number increases, the axial length $L_{z}$ of the microcapsule extends and becomes larger than the capillary diameter highlighting a long lubrication film. This is amplified for $\beta>1$ (Fig. 5-d). The local confinement under flow $L_{r} / R$ is so the relevant parameter in this limit, because the thin film of thickness $e \approx R-L_{r}$ and length $L_{z}$ exerts a viscous force of about $2 \pi R \eta L_{z} V_{\text {caps }} / e$. This force is counterbalanced by the pressure difference acting on the rear and the front of the microcapsule $\pi R^{2} L_{z}(\mathrm{~d} p / \mathrm{d} z)$. Finally, the only geometrical parameter that plays a role on the equilibrium, given by $2 \eta V_{\text {caps }} \approx \mathrm{d} p / \mathrm{d} z\left(1-L_{r} / R\right)$, is the local confinement $L_{r} / R$. This analysis allows us to understand why the experimental evolution of $V_{\text {caps }} / \bar{V}$ with $L_{r} / R$ corresponds perfectly with the analytical solutions obtained for rigid sphere on a large range of confinement [56], $\beta$ ranging from 0.1 to 0.9 . These results match also asymptotic analytical results [32] in the limit $\beta \rightarrow 1$ :

$$
\frac{V_{\text {caps }}}{\bar{V}}=\frac{1}{1-\frac{4}{3} \frac{1-\beta}{\beta}+\frac{46}{15}\left(\frac{1-\beta}{\beta}\right)^{2}} \text { for } \beta \rightarrow 1
$$

Finally, the local confinement $L_{r} / R$ allows us to find the same evolution of the velocity of the microcapsule $V_{\text {caps }}$ 
as the one obtained for rigid spheres [56], albeit large axial extension $L_{z}$. This latter has counterbalanced effects on the pressure force pushing the capsule and the viscous stress in the thin film between the capsule and the capillary.

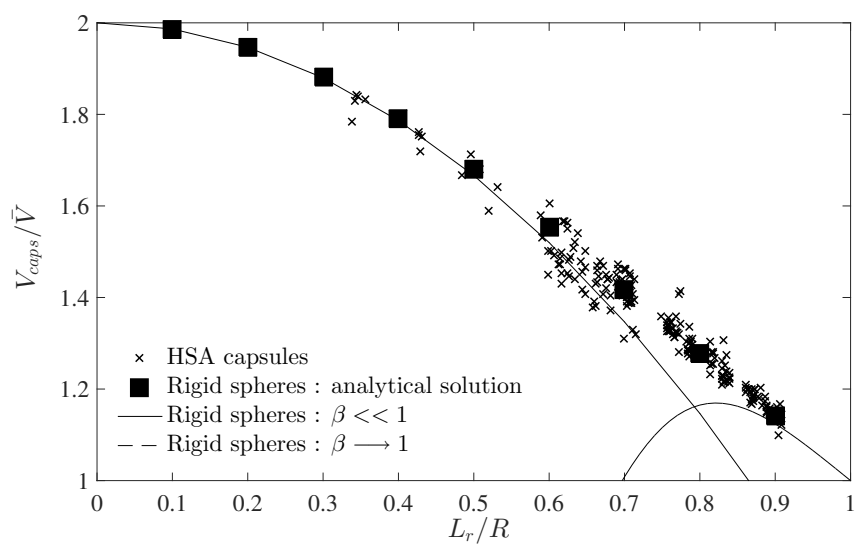

FIG. 6. Microcapsule velocity $V_{\text {caps }}$ normalized by the mean flow velocity $\bar{V}$ as a function of the local confinement $L_{r} / R$. Experimental results are compared with asymptotic analysis $[31,32]$ and analytical results [56] for hard spheres with radii equal to $L_{r}$.

\section{FLOW AROUND MICROCAPSULES}

In this section, we focus on the perturbed flow due to the presence of a microcapsule moving along the capillary in its stationary state. We report the experimental results obtained by PTV and extract the essential facts. We compare the results with numerical simulations at constant shape provided by experiments with no-slip condition (rigid body simulations)[49-54]. This is relevant as the capsules have an axisymmetrical shape in the stationary state. In all generality, the flow profile is noted $\mathbf{V}(r, z)=\left(v_{r}, v_{z}\right)$ in the reference frame of the microcapsule.

\section{A. recirculation region}

In the reference frame of the microcapsule, the fluid moves forward in the central region and backward close to the channel wall (Fig. 4). The velocity of the fluid in the axial direction of the capillary $v_{z}(r, z)$ shows a sign inversion between these two regions. At the front and the rear of the soft object, the flow presents two recirculation regions characterized by a non-nul radial component of the flow velocity $v_{r}(r, z)$ and the presence of stagnation rings on the rear and the front of the microcapsule (Fig. $4,7)$. The size of these inversion zones is defined by $\lambda(z)$ the radius for which $v_{z}(r, z)$ equals zero, i.e. $v_{z}(\lambda, z)=$ 0 . Far from the microcapsule, $\lambda$ is deduced analytically by subtracting the velocity of the microcapsule to the velocity profile of a Poiseuille flow:

$$
\lambda(z)=R\left(1-\frac{V_{\text {caps }}}{2 \bar{V}}\right)^{1 / 2} \text { for } z \rightarrow \pm \infty
$$

The numerical and experimental evolution of $\lambda$ with the axial direction $z$ is compared in Fig. 7. Note that the experimental flow field has been interpolated to obtain a better precision on the position of $\lambda$. As expected $\lambda$ is constant far from the microcapsule with only $9 \%$ of difference between experimental and theoretical value (equation 7). That is in agreement with the integration experimental results over the depth of field of the objective (see section IID).
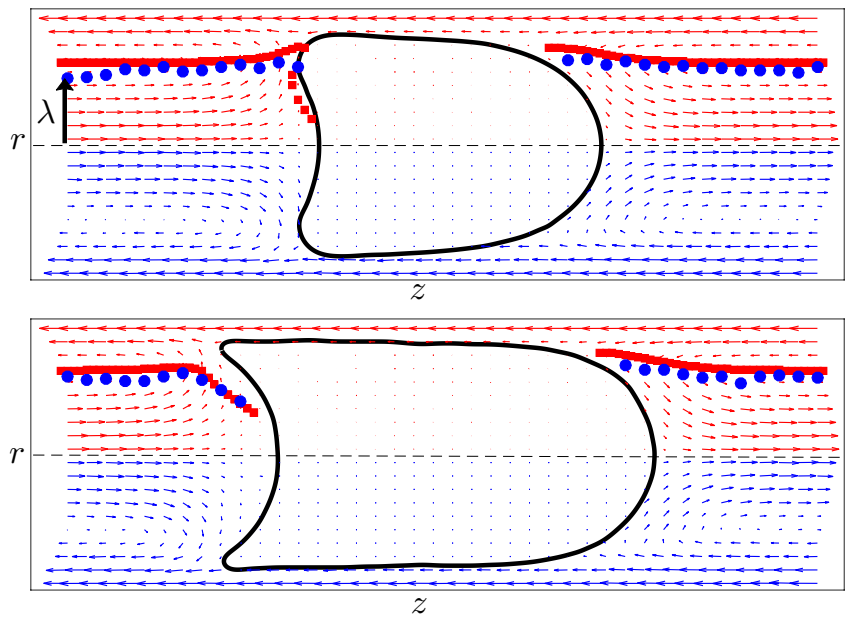

FIG. 7. Velocity field and recirculation regions determined experimentally and numerically for two different microcapsules. Experimental (blue) and numerical (red) results are shown on the lower and upper half part of the capillary tube respectively. The size of the inversion zone $\lambda(z)$ is defined as the distance from the centerline to $v_{z}(\lambda, z)=0$. Blue circles shows $\lambda$ obtained from experiments while red squares from numerical simulations.

The size of the image is $1162 \times 403 \mu \mathrm{m}^{2}$. Top: $a=195 \mu \mathrm{m}$, $\beta=0.95, \bar{V}=4.04 \mathrm{~mm} / \mathrm{s}, V_{\text {caps }}=4.98 \mathrm{~mm} / \mathrm{s}, C a=0.01$ ; Bottom: $a=231 \mu \mathrm{m}, \beta=1.10, \bar{V}=13.72 \mathrm{~mm} / \mathrm{s}, V_{\text {caps }}=$ $16.44 \mathrm{~mm} / \mathrm{s}, C a=0.018$.

Close to the microcapsule, the radius of the inversion zones $\lambda(z)$ changes notably (Fig. 7). The regions of positive (red) and negative (blue) values of the flow velocity in the axial direction $v_{z}$ are also highlighted in Fig. 8 with numerical simulations done for the same microcapsule $(\beta=0.95)$ showing 'bullet-like' and 'parachute-like' shapes as its velocity $V_{\text {caps }}$ increases. At the front of the microcapsule, the curvature of the membrane being always the same whatever the confinement or the capillary number are, the radius of the inversion zone $\lambda$ exhibits the same behavior with only one recirculation region and one stagnation ring. This flow characteristic is captured both numerically and experimentally by PTV and was also observed for drops or bubbles [30, 33, 36, 37, 46, 47]. 

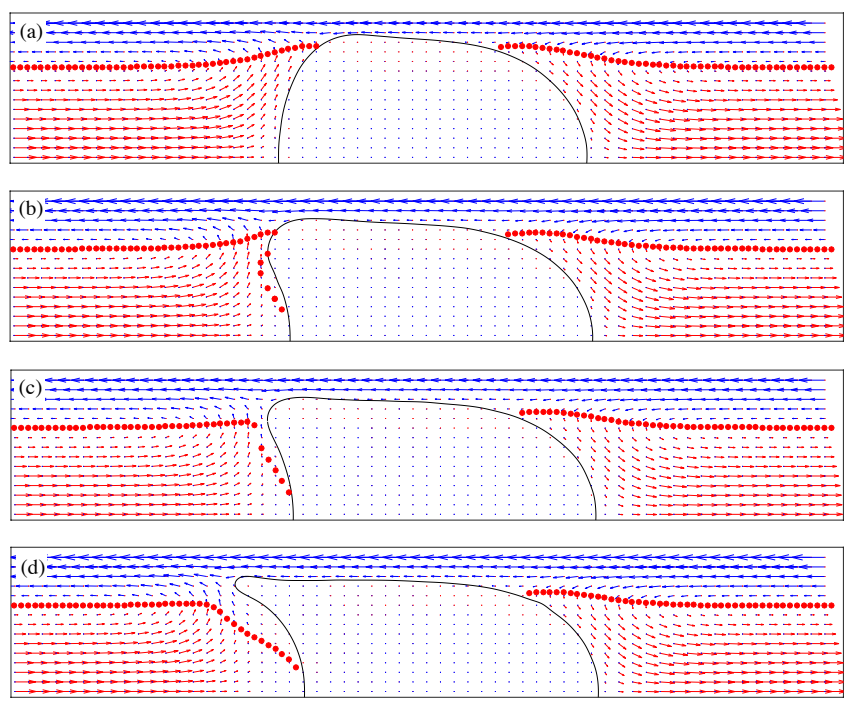

FIG. 8. Numerical simulations of the velocity field around the same microcapsule $(a=195 \mu \mathrm{m}, \beta=0.95)$ for different mean flow velocities $\bar{V}$ and position of the inversion zone $\lambda$ (red circles). Red and blue arrows show positive and negative axial flow velocity $v_{z}$ respectively. From top to bottom $\bar{V}$ equal to $1.95 \mathrm{~mm} / \mathrm{s} ; 4.04 \mathrm{~mm} / \mathrm{s} ; 6.68 \mathrm{~mm} / \mathrm{s}$ and $13.92 \mathrm{~mm} / \mathrm{s}$. Note : same capsule than row c in figure 4.

In contrast, at the rear of the microcapsule, it is noteworthy to see that the position as well as the number of stagnation rings of the capsule vary with the shape (Fig. $7 \& 8$ ). The shape of these recirculation domains is closely related to the variations of curvature of the membrane in this region. Three stagnation rings on the microcapsule can be observed when the rear curvature starts to invert and a small additional recirculation region is generated in the vicinity of the curvature inversion (Fig. 7-top \& 8-b) and $\lambda$ can have multiple values for the same abscissa $z$. The lack of particles close to the membrane does not provide enough information to capture experimentally these small variations of $\lambda$. Increasing again the curvature of the rear, we observe experimentally and numerically that the two recalculations regions finally collapse in one (Fig. 7-bottom \& 8-c). For high velocity, the backward region penetrates inside the inverted rear of the microcapsule to almost reach the axis of the channel. Thus only one stagnation ring remains close to the centerline (Fig. 8-d).

\section{B. Perturbation region}

The presence of a single microcapsule in a Poiseuille flow perturbs the flow by creating recirculation regions at the rear and the front (Fig. 7). We determine the effects of the local confinement on the amplitude and the spatial extent of this perturbation. In this way, the averaged radial velocity $<\left|v_{r}\right|>$ along the capillary radius is calculated as a function of the axial

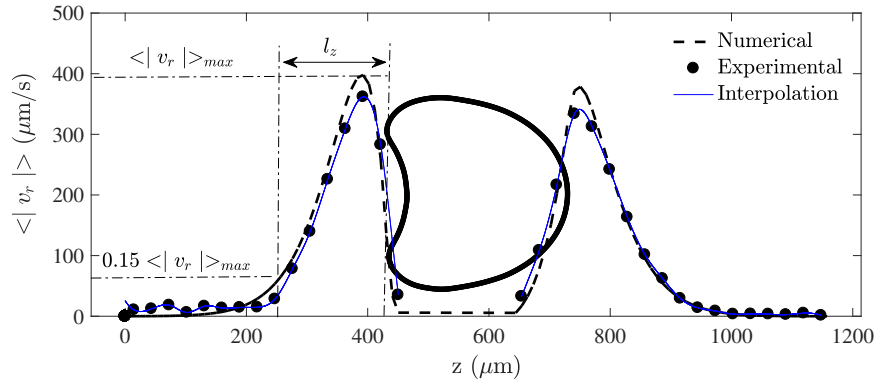

FIG. 9. Front and rear perturbation regions are calculated numerically and experimentally from the mean of the radial velocity $<\left|v_{r}\right|>$ for each cross section $z$. These perturbations are characterized by the local maxima of the mean radial velocity $<\left|v_{r}\right|>_{\max }$ and the length $l_{z}$ between one microcapsule end to the threshold $0.15<\left|v_{r}\right|>_{\max }$. The microcapsule is the same as presented in Fig. 3

position z. Experimental and numerical results show that $<\left|v_{r}\right|>$ vanishes far from the microcapsule and two distinct perturbation zones appear close to it (Fig. 9). To quantify the perturbation regions, the maximum amplitude of $<\left|v_{r}\right|>$, called $<\left|v_{r}\right|>_{\max }$ is determined at the rear and also to the front of the microcapsule. The experimental data are previously smoothed by a local parabolic interpolation. Then we measure the distance $l_{z}$ from one end of the capsule to $<\left|v_{r}\right|>=0.15<\left|v_{r}\right|>_{\max }$ (Fig. 9) at the front and the rear. This cut-off value was chosen to slightly exceed the level of noise of experimental data.

The dimensionless amplitude of the perturbation $<1$ $v_{r} \mid>_{\max } / 2 \bar{V}$ increases linearly with the local confinement $L_{r} / R$ (Fig. 10). Again, experimental data are underestimated of about $9 \%$ compared to numerical ones due to the depth of field of the objective. For moderate confinements $\left(L_{r} / R \approx 0.7\right)$, the amplitude is the same for both sides of the microcapsule, i.e. about $12.4 \%$ of the mean velocity of the Poiseuille flow. However, the amplitude of the rear perturbation region increases more steeply than the front one to reach up to 0.09 compared to 0.075 for a local confinement $L_{r} / R=0.9$.

While the amplitude varies with $L_{r} / R$, the length of the perturbation $l_{z}$ shows no variations neither with the capillary number nor with the local confinement $L_{r} / R$. $l_{z}$ is smaller upstream than downstream. For numerical simulations $l_{z} / R$ is equal to $0.87 \pm 0.02$ for the rear region and $0.70 \pm 0.02$ for front regions whatever $C a$ and $a / R$. These values are supported by experiments: $l_{z} / R$ equal to $0.87 \pm 0.03$ for rear regions and $0.77 \pm 0.04$ for the front. In figure 9 we notice that the amplitude of the perturbation zone abruptly increases from the capsule rear while it slowly increases and partially covers its front. As $l_{z}$ is measured from both ends of the capsule, this implies that $l_{z}$ is smaller at the front than at the rear of the capsule. Our results are in good agreement with theoretical developments for rigid spheres [57]. A distance of about 



FIG. 10. Maximum of the average of the radial velocity $<1$ $v_{r} \mid>_{\max } / 2 \bar{V}$ at the rear (top) and the front (bottom) of the microcapsule as a function of the local confinement $L_{r} / R$. The amplitude of the perturbation increases with the local confinement whereas its axial extent $l_{z}$ is constant.

$R$ up and downstream is expected for the velocity of a cylindrical Poiseuille flow to recover unperturbed when locally disturbed.

\section{CONCLUSION}

The velocity field around microcapsules in capillary tubes of comparable size has been explored. PTV measurements of experimental observations were compared successfully with numerical simulations developed from experimental microcapsule profile and velocity. Three aspects of the velocity fields have been investigated: the microcapsule velocity, the recirculation and disturbance regions. Some aspects of the flow are similar to the case of rigid spheres, drops or bubbles, whereas when the curvature of the rear of the microcapsule is inverted, the topology of the recirculation region has never been observed for such elongated shapes with no-slip condition at the interface due to the specificity of microcapsules.

The microcapsule velocity $V_{c}$ is found to depend only on its radial extension $L_{r} / R$ and unexpectedly not on its axial length $L_{z}$ whereas it varies in our experiment up to four times its radial extension (Fig. 5 -d and $C a=0.018$ ). This is explained by the simultaneous increasing pressure drop and shear force in the thin film governed by lubrication. For this reason, the experimental capsule velocities are in good agreement with the analytical expression for confinement $0<L_{r} / R<0.6$ and numerical simulation for $L_{r} / R>0.65$, both considering the motion of a rigid sphere $[31,32,56]$.

Since the microcapsule velocity varies between the mean flow rate and the maximal velocity of Poiseuille flow, the flow exhibits a front and back recirculation regions. Similarly to drops and bubbles[30, 33, 36, 37, 46, 47], two stagnation rings were observed experimentally at the rear and the front of the microcapsule surface for low capillary numbers. With the rising of the capillary number, the radius of the ring at the front does not change while experimental results show a strong decreasing of the radius at the rear. This is due to the large change of shape at the rear. These are in agreement with numerical studies even if for moderate capillary numbers, the picture is more complex with the emergence of three stagnation rings at the rear (Fig. 8-b).

In addition to the recirculation regions, the velocity field is disturbed over a given distance which is a key parameter when multiple objects interact hydrodynamically. This is a preliminary before understanding microcapsules interaction in a Poiseuille flow. Indeed, drops, vesicles and red blood cells can assemble in a cluster in a Poiseuille flow as shown numerically $[28,58,59]$ for example. We expect a similar effect with microcapsules. If the range of perturbed region does not vary with the capillary number and confinement, the amplitude of perturbed flow varies linearly with the confinement and more steeply for the rear part. Such a variation should affect the interaction between two microcapsules moving along a channel.

\section{AKNOWLEDGMENTS}

This work has benefited of financial support from the ANR CAPSHYDR (11-BS09-013- 02), from Labex MEC (ANR-11-LABX-0092), from A*MIDEX (ANR-11IDEX-0001-02) and from CNES. This work was granted access to the HPC resources of Aix-Marseille Université financed by the project Equip@Meso (ANR-10-EQPX29-01).
[1] M. N. Singh, K. S. Hemant, M. Ram, and H. G. Shivakumar, Res. Pharm. Sci. 5, 65 (2010).

[2] F. Edwards-Lévy, "Serum albumin: structure, functions and health impact," (Nova Science Publishers, New-
York, 2011) Chap. Microparticles drug delivery systems based on serum albumin, pp. 69-100.

[3] S. Banquet, E. Gomez, L. Nicol, F. Edwards-Lévy, J.-P. Henry, R. Cao, D. Schapman, B. Dautreaux, F. Lalle- 
mand, F. Bauer, et al., Circulation 124, 1059 (2011).

[4] T. Chang, Journal of internal medicine 253, 527 (2003).

[5] Y. Long, C. Liu, B. Zhao, K. Song, G. Yang, and C.-H. Tung, NPG Asia Materials 7, e148 (2015).

[6] M.-C. Andry, F. Edwards-Lévy, and M.-C. Lévy, International journal of pharmaceutics 128, 197 (1996).

[7] D. J. McClements, E. A. Decker, Y. Park, and J. Weiss, Critical Reviews in Food Science and Nutrition 49, 577 (2009).

[8] D. Z. Gunes, M. Pouzot, M. Rouvet, S. Ulrich, and R. Mezzenga, Soft Matter 7, 9206 (2011).

[9] C. de Loubens, J. Deschamps, M. Georgelin, A. Charrier, F. Edwards-Levy, and M. Leonetti, Soft matter 10, 4561 (2014).

[10] I. Koleva and H. Rehage, Soft Matter 8, 7672 (2012).

[11] K. Chang and W. Olbricht, Journal of Fluid Mechanics 250, 609 (1993).

[12] K. Chang and W. Olbricht, Journal of Fluid Mechanics 250, 587 (1993).

[13] C. de Loubens, J. Deschamps, G. Boedec, and M. Leonetti, Journal of Fluid Mechanics 767, R3 (2015).

[14] F. Risso and M. Carin, Phys. Rev. E 69, 061601 (2004).

[15] I. Koleva and H. Rehage, Soft Matter 8, 3681 (2012).

[16] A. Walter, H. Rehage, and H. Leonhard, Colloid and Polymer Science 278, 169 (2000).

[17] F. Risso, F. Collé-Paillot, and M. Zagzoule, Journal of fluid mechanics 547, 149 (2006).

[18] Y. Lefebvre, E. Leclerc, D. Barthès-Biesel, J. Walter, and F. Edwards-Lévy, Physics of Fluids 20, 123102 (2008).

[19] G. Dawson, E. Haner, and A. Juel, Procedia IUTAM 16, 22 (2015).

[20] G. Pieper, H. Rehage, and D. Barthès-Biesel, Journal of colloid and interface science 202, 293 (1998).

[21] C. de Loubens, J. Deschamps, F. Edwards-Lévy, and M. Leonetti, Journal of fluid mechanics $\mathbf{1 0 . 1 0 1 7 / j f m . 2 0 1 5 . 7 5 8 ~ ( 2 0 1 6 ) . ~}$

[22] M. P. Keh, J. Walter, and L. G. Leal, Physics of Fluids 26, 111903 (2014).

[23] P. Dimitrakopoulos, J. Fluid Mech. 745, 487 (2014).

[24] A. Yazdani and P. Bagchi, Journal of Flluid Mechanics 718, 569 (2013).

[25] C. Dupont, A.-V. Salsac, D. Barthès-Biesel, M. Vidrascu, and P. L. Tallec, Physics of Fluids 27, 051902 (2015).

[26] P.-Y. Gires, A. Srivastav, C. Misbah, T. Podgorski, and G. Coupier, Physics of Fluids 26, 013304 (2015).

[27] W. L. Olbricht and D. M. Kung, Journal of colloid and interface science 120, 229 (1987).

[28] P. Janssen, M. Baron, P. Anderson, J. Blawzdziewicz, M. Loewenberg, and E. Wajnryb, Soft Matter 8, 7495 (2012).

[29] G. Tomaiuolo, L. Lanotte, G. Ghigliotti, C. Misbah, and S. Guido, Physics of Fluids (1994-present) 24, 051903 (2012).

[30] G. Taylor, Journal of Fluid Mechanics 10, 161 (1961).

[31] H. Brenner, Journal of Fluid Mechanics 43, 641 (1970).
[32] P. M. Bungay and H. Brenner, International Journal of Multiphase Flow 1, 25 (1973).

[33] G. Hetsroni, S. Haber, and E. Wacholder, Journal of Fluid Mechanics 41, 689 (1970).

[34] A. Nadim and H. A. Stone, Studies in Applied Mathematics 85, 53 (1991).

[35] L. Leal, Annual Review of Fluid Mechanics 12, 435 (1980).

[36] B. Ho and L. Leal, Journal of Fluid Mechanics 71, 361 (1975).

[37] M. Martinez and K. Udell, Journal of fluid mechanics 210, 565 (1990).

[38] R. Bruinsma, Physica A: Statistical Mechanics and its Applications 234, 249 (1996).

[39] V. Vitkova, M. Mader, and T. Podgorski, EPL (Europhysics Letters) 68, 398 (2004).

[40] G. Danker, P. M. Vlahovska, and C. Misbah, Physical review letters 102, 148102 (2009).

[41] G. Coupier, A. Farutin, C. Minetti, T. Podgorski, and C. Misbah, Physical review letters 108, 178106 (2012).

[42] A. Farutin and C. Misbah, Physical Review E 84, 011902 (2011).

[43] T. Secomb, R. Skalak, N. Özkaya, and J. Gross, Journal of Fluid Mechanics 163, 405 (1986).

[44] C. Quéguiner and D. Barthès-Biesel, Journal of Fluid Mechanics 348, 349 (1997).

[45] J. T. Schwalbe, F. R. Phelan Jr, P. M. Vlahovska, and S. D. Hudson, Soft Matter 7, 7797 (2011).

[46] W. Olbricht, Annual review of fluid mechanics 28, 187 (1996).

[47] C. Pozrikidis, Journal of fluid mechanics 237, 627 (1992).

[48] J. Gubspun, P.-Y. Gires, C. de Loubens, D. BarthèsBiesel, J. Deschamps, M. Georgelin, M. Leonetti, E. Leclerc, F. Edwards-Lévy, and A.-V. Salsac, Colloid and Polymer Science 10.1007/s00396-016-3885-8 (2016).

[49] C. Pozrikidis, Cambridge University Press (1992).

[50] C. Pozrikidis, Modeling and simulation of capsules and biological cells (CRC Press, 2003).

[51] R. Trozzo, G. Boedec, M. Leonetti, and M. Jaeger, Journal of Computational Physics 289, 62 (2015).

[52] G. Boedec, M. Leonetti, and M. Jaeger, Journal of Computational Physics 230, 1020 (2011).

[53] G. Boedec, M. Jaeger, and M. Leonetti, Journal of Fluid Mechanics 690, 227 (2012).

[54] G. Boedec, M. Jaeger, and M. Leonetti, Physical Review E 88, 010702 (2013).

[55] F. P. Bretherton, J. Fluid Mech. 10, 166 (1961).

[56] H. Wang and R. Skalak, Journal of Fluid Mechanics 38, 75 (1969).

[57] N. Liron and R. Shahar, Journal of Fluid Mechanics 86, 727 (1978).

[58] J. L. McWhirter, H. Noguchi, and G. Gompper, Proceedings of the National Academy of Sciences 106, 6039 (2009).

[59] G. Ghigliotti, H. Selmi, L. El Asmi, and C. Misbah, Physics of Fluids (1994-present) 24, 101901 (2012). 